\title{
PROYECCIONES DE LA FUERZA DE TRABAJO DE MÉXICO*
}

\author{
Donald B. KeEsing y Alan S. ManNe** \\ Banco Internacional de Reconstrucción y Fomento
}

\section{INTRODUCCIóN}

En este documento se describen las proyecciones de los recursos humanos dentro de un modelo dinámico multisectorial de la economía mexicana. El modelo (que por brevedad llamaremos DINAMICO), incluye no sólo restricciones sobre insumos y productos, inversiones de capital y comercio internacional, sino también restricciones sobre la oferta y la demanda de cinco clases de calificación de la fuerza de trabajo. La estructura formal del modelo aparece descrita en un informe separado. ${ }^{1}$

DINÁMICO no ha sido diseñado para la planeación de los recursos humanos. Más bien pretende proporcionar proyecciones macroeconómicas junto con precios de eficiencia de los factores clave de la producción. El modelo incorpora ciertos aspectos del potencial de México en cuanto a recursos humanos: coeficientes de insumos de trabajo, formación de capital mediante la educación y sustitución de calificaciones. Los aspectos menos sujetos a cuantificación y a control premeditado - por ejemplo, los cambios en los patrones individuales de conducta, las actitudes y las estructuras de clase- han sido omitidos por necesidad.

* Versión revisada de un documento presentado ante el Segundo Congreso Mundial de la Sociedad de Econometría, Cambridge, Inglaterra, septiembre de 1970. Esto formará un capítulo en un libro cuyo título provisional será Project Decisions and Multi-level Planning: Case Studies in Mexico. La investigación fue patrocinada por el Centro de Investigaciones para el Desarrollo del Banco Internacional de Reconstrucción y Fomento. Los hechos específicos, los métodos de análisis y las conclusiones son de la exclusiva responsabilidad de los autores. El título original de este artículo es "Manpower Projections for Mexico" y la traducción al español es de A. García Rocha.

** Por haber recibido ayuda de parte de varias personas en varias fases de este estudio, nos gustaría expresar nuestro agradecimiento a todos aquellos que han contribuido con su consejo y ayuda. Se debe agradecimiento especial a las siguientes personas: Lic. Leopoldo Solís, Dr. Luciano Barraza, Ing. Víctor Ramírez Izquierdo, Lic. Antonio Suárez McAuliffe, Lic. Saúl Trejo y Lic. Alfredo Santos Arenas (del Banco de México); Lic. Gustavo Cabrera Acevedo, Lic. José B. Morelos, Ing. Luis Unikel y Sra. Clara J. de Bialostozky (de El Colegio de México); Lic. Gerardo Bueno (de Nacional Financiera); profesor Janos Kornai (Academia Húngara de Ciencias); Dr. Louis Goreux (BIRF); y profesores Mordecai Kurz y Clark W. Reynolds y Sr. Richard Inman (de la Universidad de Stanford).

1 Ver el memorándum 70-13, "A Dynamic Multi-sector, Multi-skill Model for Mexico, 1968-1980", obtenible del Centro de Investigación del Desarrollo. Banco Internacional de Reconstrucción y Fomento, 1818 H. Street, N. W. Washington, D. C. 20433 . 
La calidad de los datos manejados es dispar y el modelo tiene simplificaciones inevitables en comparación con la realidad de la economía mexicana. Por consiguiente, debe tenerse mucha precaución al utilizar los resultados. Debe confiarse más en las proyecciones agregativas y en las tendencias a largo plazo que en los detalles anuales o los sectoriales.

Los resultados son compatibles con el crecimiento del producto bruto interno a una tasa anual de $7 \%$ entre 1968 y 1980 . Resulta que la mano de obra calificada y la no calificada, el capital de inversión $\mathrm{y}$ el comercio exterior son todos factores escasos que limitan en forma conjunta esta tasa de crecimiento.

Tal vez el resultado que despierta más controversia es aquel relacionado con la fuerza de trabajo no calificada. De acuerdo con DINAMICO, si el crecimiento económico continúa durante los años setenta, será óptimo sustituir mano de obra agrícola no calificada por otros factores productivas. Como resultado, el número absoluto de ocupación agrícola probablemente permanecerá aproximadamente constante. Este hallazgo descansa en varios supuestos optimistas: 1) existe una percepción perfecta del futuro - por lo tanto, no existen interrupciones ni perturbaciones del proceso de crecimiento- y ninguna imperfección del mercado impide la utilización eficiente de los recursos; 2) el crecimiento rápido se proyecta sobre la oferta de factores productivos complementarios: el capital físico y la mano de obra calificada, entre otros -ésta es condición necesaria para la expansión de la demanda de mano de obra no calificada fuera de la agricultura tradicional ; 3) fuera de la agricultura, se supone que la productividad incorporada del trabajo crecerá en forma sostenida, aunque no dramática : $3.5 \%$ al año en la industria y $1.5 \%$ en los servicios, y 4 ) tal vez el supuesto más discutible que hacemos es que no se ha tomado en cuenta una reserva inicial grande de desempleo disfrazado. No conocemos ninguna medida confiable del tamaño de tal reserva, y sospechamos que en México la pobreza está relacionada con la baja productividad, no con el desempleo propiamente dicho.

Estas proyecciones de la demanda de mano de obra contrastan con la tendencia observada de un desempleo creciente en otros países en desarrollo.2 Nuestros resultados sugieren que puede alcanzarse un punto de inflexión en el desarrollo económico, después del cual los cambios estructurales continuados comienzan a crear escasez de fuerza de trabajo no calificada. Existen razones para creer que esto podría suceder, porque México ya ha pasado por un proceso de desarrollo económico sostenido y largo. En comparación, hasta los años treinta, la modernización parece claramente haber desplazado personal de las ocupaciones tradicionales con mayor velocidad que con la que creó empleos nuevos. ${ }^{3}$

\section{EL ANÁLISIS DE LOS RECURSOS HUMANOS EN DINAMICO}

DINAMICO es un modelo de programación lineal que contiene 244

2 Ver Frank (1968), Oshima (1968) y Thorbecke (1970), para citar solamente unas cuantas referencias de una literatura enorme y alarmante.

3. Ver Keesing (1969). 
hileras. Los recursos escasos se han de asignar entre los sectores y las actividades en cada período de modo tal de maximizar el crecimiento del consumo, sujeto a la condición de que los incrementos del consumo deben crecer al $8 \%$ anual después de 1971. (Esto implica que la tasa de crecimiento del consumo aumentará en el tiempo, acercándose en forma asintótica a $8 \%$.) El consumo per capita de cada rubro en cada año se relaciona linealmente (pero no proporcionalmente) con el consumo per capita de todos los rubros combinados. El crecimiento económico está también sujeto a una diversidad de otras restricciones y requisitos de consistencia, por ejemplo, relaciones insumo-producto, requisitos de inversión para el crecimiento de la capacidad, y restricciones sobre la balanza de pagos. Siguiendo el criterio de agregación utilizado en el Banco de México (1970 b), las actividades económicas de producción se clasifican en 15 sectores.

El modelo se enfoca sobre cuatro puntos representativos de tiempo, separados cada uno por tres años —1971, 1974, 1977 y 1980-; el año 1968 sirve de base estadística. Se incluye un quinto período (1983) para reducir los "efectos de horizonte" sobre la calificación de la mano de obra. Los períodos sucesivos se identifican mediante el índice $t$, el cual es cero para $1968, t=1$ para 1971 y así sucesivamente.

Los principales recursos escasos se han definido como la producción y la capacidad de cada sector en cada período, la disponibilidad de mano de obra en cinco clases de calificación, la inversión de capital, y el ingreso de divisas. A partir del año base estadístico, los incrementos de capital físico y trabajo calificado se producen en forma endógena a un costo de recursos escasos. Se incluyen también actividades (exportaciones industriales aditivas, sustitución de importaciones y préstamos del exterior) para ampliar la oferta de divisas a un costo marginal elevado - por encima de los límites permitidos por cuotas superiores especificas sobre el crecimiento de las exportaciones en cada sector. La oferta total de trabajo se proyecta de manera exógena. El trabajo no calificado se considera como un factor primario, pero la "mezcla" de calificaciones * puede ser mejorada mediante actividades dirigidas a la formación del capital humano.

Para simplificar, se supone que permanecerán disponibles las capacidades física y de fuerza de trabajo utilizadas en cada sector en el año base (1968) y que, salvo en el sector agrícola, continuarán elaborando el mismo producto en todos los años subsecuentes. De este modo, el problema de programación consiste en asignar los incrementos de producción y de crecimiento de la fuerza de trabajo, tomando en cuenta no sólo las relaciones de insumo-producto actuales, sino también relaciones intertemporales, según las cuales la formación de capital físico y humano debe preceder a los aumentos de la producción.

La demanda de mano de obra se ha determinado con base en coeficientes de trabajo por unidad de producto rígidos en cada sector y cada clase de calificación. En los sectores no agrícolas, la productividad del trabajo crece a través del progreso técnico incorporado que afecta solamente los nuevos incrementos de la capacidad. En el margen, cada sector productivo se caracteriza por una mezcla porcentual

* Skill-mix (T.) 
constante de calificaciones y una norma total de insumo de trabajo decreciente.

En el caso de la agricultura, se ha especificado la posibilidad de seleccionar de entre cuatro técnicas de producción alternativas: mecanizada, moderna, tradicional y de subsistencia - cada una de las cuales tiene sus propios coeficientes de insumo-producto constantes. Se supone que el capital y el trabajo dedicados a la agricultura tradicional en el año base (1968) pueden desplazarse subsecuentemente hacia la agricultura moderna o la mecanizada, pero que dentro de un año existe un límite a la tasa de modernización. Como resultado, la agricultura tradicional se puede reducir en forma sostenida, pero no puede desaparecer sino hasta después de 1980.

En la transición inicial de 1968 a 1971, el crecimiento del trabajo calificado y el no calificado se estima exógenamente con base en sus tendencias demográficas y educativas. En el caso de los científicos y los ingenieros, la rigidez de la oferta resultante se prolonga hasta 1974, debido al rezago de seis años que toma su adiestramiento. Los incrementos subsecuentes de la oferta de fuerza de trabajo se toman como parcialmente exógenos y parcialmente endógenos a través de las actividades educativas. La fuerza de trabajo de estudiantes y maestros se considera como el único insumo de las actividades endógenas de formación de capital humano.

Para ayudar a atenuar las rigideces de la oferta de fuerza de trabajo, el modelo incluye actividades de sustitución de calificación, que permiten la sustitución para evitar estrangulamientos a corto plazo en cada una de las tres categorías de calificación (aquellas basadas en la educación). En cada caso, la mano de obra de la categoría de calificación siguiente superior puede sustituir a la calificación faltante. Los costos toman la forma de productividad disminuida de la fuerza de trabajo en la fuerza de trabajo asociada. Estas actividades de sustitución a corto plazo se suponen disponibles sólo durante los dos períodos iniciales.

En todos los períodos permanecen abiertas otras dos posibilidades de sustitución. Primero, el trabajo puede ser desplazado de la clase 5 (trabajo agrícola no calificado) a la clase 4 (trabajo ordinario fuera de la agricultura) al costo de insumos adicionales de servicios urbanos. De este modo, ya sea directamente o a través de la educación, en cada período el trabajo puede elevarse de las categorías de calificación menores a las mayores, pero siempre sujeto a un costo. Segundo, el trabajo puede ser desplazado libremente a las clases de calificación inferior.

Si un trabajador no puede encontrar empleo en ningún lado, puede dedicarse a una actividad agrícola de subsistencia que no requiera insumos interindustriales ni adiciones netas al acervo de capital.

A diferencia de las condiciones finales en cuanto a la formación de capital físico, no se prevé el adiestramiento de fuerza de trabajo calificada adicional en 1983, para su uso en años subsecuentes. Durante el año final, la fuerza de trabajo económicamente activa se asigna sólo a los sectores que producen bienes y servicios. Se necesita investigación ulterior para llegar a una especificación del modelo que reduzca los "efectos de horizonte" en el caso de los recursos humanos: 


\section{Definiciones de las categorías de calificación}

La fuerza de trabajo se divide en cinco clases de calificación, denotadas mediante $s=1,2,3,4$ y 5 . Éstas se definen como:

1. Ingenieros y científicos.

2. Otros trabajadores profesionales y técnicos.

3. Trabajadores administrativos y de oficina.

4. Trabajadores manuales y de ventas, no agrícolas.

5. Trabajadores agrícolas no calificados.

La oferta de 1960 se ha estimado principalmente con base en los datos ocupacionales del censo de población. En cuanto a 1968, el crecimiento de las clases 1,2 y 3 se basa en una proyección de la demanda de las mismas ocupaciones.

1. Científicos e ingenieros se define como ingenieros, químicos, otros científicos naturales, agrónomos y veterinarios, con o sin título, pero con adiestramiento universitario completo o su equivalente.

2. Otros profesionistas y trabajadores técnicos comprende todos los tipos de trabajadores clasificados como profesionistas o técnicos según los censos de población de 1950 y 1960, excepto los científicos e ingenieros de la definición anterior. En la práctica, ésta es una categoría heterogénea con amplia variedad de antecedentes educativos - desde animadores iletrados hasta médicos de largo adiestramiento y profesores universitarios.

3. Trabajadores administrativos $y$ de oficina se definen, en 1960 , mediante un conjunto de categorías ocupacionales censales, incluidos todos los trabajadores de oficina, una décima parte de todos los trabajadores de ventas y el personal administrativo y directivo de las empresas más grandes. ${ }^{4}$

Los trabajadores restantes se han dividido entre la cuarta y la quinta categoría dependiendo sólo de que estén o no dedicados a la agricultura. Al seleccionar las categorías de clasificación ha sido imposible distinguir entre trabajadores calificados, semicalificados o no calificados. También ha sido imposible tomar en cuenta en forma explícita el mejoramiento de los trabajadores hacia categorías de calificación superiores a través del adiestramiento en el trabajo en lugar de educación formal. Estas omisiones se originan en la ausencia casi total de datos.

Esta clasificación en cinco categorías sobresimplifica la realidad. No obstante, es una transacción que mantiene a DINÁMICO dentro de un tamaño manejable y saca ventaja de los tipos de información disponible, tanto en México como en otros países. Para otros propósitos puede resultar esencial definir las categorías de trabajo con mucho mayor detalle que como se hace aquí.

4 El personal directivo y administrativo incluye aquellos definidos por el censo como personal directivo no agrícola, los propietarios agrícolas y los grandes empresarios dentro de la categoría patrón-empresario-empleador, y se toman en cuenta los operadores-propietarios de los sectores de construcción y transporte. 


\section{Oferta de Mano dE ObRa PROYectada EN forma EXógena}

En el cuadro 1 aparecen resumidas nuestras proyecciones exógenas de fuerza de trabajo disponible en cada período previo a los efectos de las actividades endógenas educativas y de sustitución de calificaciones. Las proyecciones de la población y la fuerza de trabajo total se basan en estimaciones demográficas y toman en cuenta cambios en la estructura por edad y sexo de la población. 5

Después de 1971 , se considera un crecimiento del $2 \%$ anual de la clase 5 de mano de obra ante la ausencia de diferencia alguna entre los salarios de la mano de obra no calificada urbana y rural. En vista de que la tasa de crecimiento natural de la población es al menos tan alta en el medio rural como en el resto, la clase 5 crecería por lo menos al $3.3 \%$ si no hubiera migración rural-urbana. El cuadro $1 \mathrm{im}$ plica que alrededor del $40 \%$ del incremento anual de la fuerza de trabajo rural se transferirá en forma automática fuera de la agricultura, aun en la ausencia de diferencias de salario. Sobre esta base, la fuerza de trabajo no agrícola crecería al $4.4 \%$ anual durante los años setenta.

Entre 1968 y 1971, las tasas de expansión de la oferta de las clases de calificación 1-3 se proyectan como una continuación de las tendencias de 1960 a 1968. Subsecuentemente, ante la ausencia de actividades endógenas de formación de calificación, se supone que las tasas de crecimiento se atenuarían en estas tres clases, y que los incrementos crecerán a partir de entonces al $4.4 \%$ anual -a la misma tasa que el total exógeno de las clases 1-4 durante los setenta. Salvo por lo que esté implícito en estas proyecciones de crecimiento, no se toma en cuenta específicamente la oferta de estudiantes aún involucrados en el sistema educativo. De hecho, éste es un recurso para acortar el argumento. En un modelo de planeación de la mano de obra más completo, sería preferible -aunque más tedioso- hacer un cálculo explícito del inventario inicial de estudiantes y maestros y de los aumentos subsecuentes en dicho inventario. Aquí si fuera óptimo asignar una intensidad positiva a las actividades de desplazamiento a niveles inferiores del trabajo $L D_{s}^{t}$ (descritas luego), esto indicaría que nuestro atajo es insatisfactorio, y que hemos dado lugar a un aumento exógeno excesivo de la oferta de la clase de calificación $s$.

En cierto momento consideramos seriamente tomar en cuenta el desempleo abierto. Sin embargo, el censo de 1960 clasificó solamente 168000 personas como desempleadas (buscadores de trabajo). Este desempleo registrado constituye sólo el $1.5 \%$ de la fuerza de trabajo, y parte de él puede haber sido desempleo transitorio. No existen estimaciones o indicadores de tendencia para los años desde $1960 .^{6}$

5 Se ha descansado en especial en la proyección II de Benítez y Cabrera (1966), pero se ha dado atención también a sus otras cifras y a las proyecciones de CELADE (1966), Tabah (1968), y a proyecciones inéditas de la fuerza de trabajo en 1985 elaboradas por José B. Morelos. Estas otras proyecciones son compatibles con nuestras estimaciones de fuerza de trabajo, excepto que Tabah prevé una disminución mayor de la tasa de crecimiento de la población y la fuerza de trabajo. Los resultados preliminares del censo de 1970 no fueron concluyentes en cuanto a si la tasa de crecimiento de la población fue mayor o menor que las estimaciones utilizadas en este trabajo.

$6 \mathrm{El}$ número de desempleados puede ponerse ante una perspectiva mejor no- 


\section{Distribución sectorial de la mano de obra SEgún clase de califica- cIÓN EN 1960 Y 1968}

Para nuestras proyecciones, no es esencial estimar la distribución sectorial de la fuerza de trabajo según clase de calificación en 1960 (último año para el cual se dispone de información censal) o en 1968. Simplemente suponemos que la fuerza de trabajo en 1968 producía, y puede continuar haciéndolo, el producto observado en ese año. La distribución sectorial en el año base 1968 es importante principalmente como guía para proyectar los coeficientes de insumo de mano de obra subsecuentes.

Las estimaciones de la fuerza de trabajo total en cada sector en 1960 (cuadro 2) se basan en datos revisados tomados del censo de población junto con una fuente que hasta ahora no había sido utilizada: una expansión sistemática de una muestra inédita del $1.225 \%$ del censo, realizada por E1 Colegio de México. En casi todos los casos, estas estimaciones concuerdan muy bien con los hallazgos desagregados encontrados en el censo de población de 1950, y con las tendencias del producto durante 1950-1960. Creemos que éstas son más exactas que otras estimaciones previas de la fuerza de trabajo sectorial de México.

La asignación de la fuerza de trabajo en 1960 (cuadro 2) ha sido lograda con ayuda de datos sobre la estructura ocupacional del censo y las muestras censales, complementados por coeficientes de mezclas de fuerza de trabajo correspondientes a otros países. Las fuentes y los métodos de estos cálculos, incluidas las definiciones operativas de las cinco clases de calificación, se encuentran disponibles por separado en otro documento mimeografiado. ${ }^{7}$ Este documento incluye también una relación de los pasos tan difíciles que seguimos para pasar del panorama de 1960 (cuadro 2) a las estimaciones comparables de 1968 (cuadro 3 ).

La estimación explícita de los cambios en 1960-1968 ha resultado útil al menos en dos aspectos. Primero, este procedimiento logra una medida de consistencia entre los supuestos del año base y los que gobiernan los requerimientos de fuerza de trabajo en años futuros. Segundo, los resultados arrojan bastante luz sobre el mecanismo de crecimiento de la productividad entre 1960 y 1968. En el proceso, ello ayuda a iluminar sesgos probables en las proyecciones de DINAMICO para 1980.

\section{Normas de INSUMO DE MANO DE OBRA No AGRÍcola}

Fuera de la agricultura, todos los requisitos de mano de obra se calcularon sobre la hipótesis de Harrod sobre cambio tecnológico neutro incorporado. Así, los aumentos de la productividad se suponen dependientes de la introducción de nuevas generaciones de bienes de capital y/o trabajo.

tando que de las 11332000 personas registradas como activas en el censo de 1960, 358000 fueron jóvenes menores de 15 años, y 463000 fueron personas de 70 y más años de edad.

7 Ver Donald Keesing, "Derivation of DINAMICO's Labor Force Skill Distribution, 1960 and 1968", Centro de Investigación del Desarrollo, Banco Internacional de Reconstrucción y Fomento, 1818 H. Street, N. W., Washington, D. C., 20433. 
Cuadro 1

México: Proyecciones de La población y de la mano de obRa según su clasificación (Antes de los efectos de actividades endógenas de formación de capital humano y de sustitución de calificación)
(miles de personas)

\begin{tabular}{|c|c|c|c|c|c|c|c|c|c|c|}
\hline & \multicolumn{8}{|c|}{$A \tilde{n} O B$} & \multirow{2}{*}{ 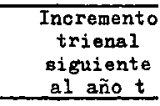 } \\
\hline & & 1950 & 1960 & 1968 & 1971 & 1974 & 1977 & 1980 & 1983 & \\
\hline \multirow{9}{*}{$\begin{array}{c}P_{\text {uerza }} \\
\text { de } \\
\text { trabajo }\end{array}$} & Perfodo $\underline{t}$ & & & 0 & 1 & 2 & 3 & 4 & 5 & \multirow{17}{*}{$\begin{array}{l}20\left(1.0444^{3 \mathrm{tz}}\right. \\
145(1.0444)^{3 \mathrm{t}}(1,1.044)^{3 \mathrm{t}}\end{array}$} \\
\hline & Poblacion & 25792 & 36004 & 47599 & 52949 & 58832 & 65192 & & 79300 & \\
\hline & cal & 19 & & 72 & 92 & 115 & 141 & 170 & 204 & \\
\hline & & 188 & 373 & 646 & 791 & 956 & 1144 & 1358 & 1601 & \\
\hline & Calificacion 3 & 545 & 915 & 1382 & 1623 & 1897 & 2209 & 2564 & 2968 & \\
\hline & Calificacion 4 & 2711 & 3916 & .5456 & 6130 & 6855 & 7656 & 8603 & 2.653 & \\
\hline & $\begin{array}{l}\text { Subtotala, } \\
\text { clases } 1-4\end{array}$ & & & 7556 & 8636 & 9823 & 11150 & 12695 & 14426 & \\
\hline & Calificacion 5 & $4.809^{a / 1}$ & $6.0911^{-1}$ & 1024 & $I_{3}^{389}$ & 7841 & 8321 & 8830 & .2370 & \\
\hline & Total, ${ }_{\text {clases }}^{\text {T-5 }}$ & 8272 & 11332 & 14580 & 16025 & 17664 & 19471 & ${ }_{21}^{2} 525$ & 23796 & \\
\hline & Pobzaa & 3. & & 3. & & 6 & 3.4 & 3. & & \\
\hline & & 6. & 8 & 8. & 7 & 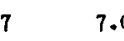 & 6.4 & 6. & & \\
\hline Taasas de & Calificacion 2 & 7. $2>3>$ & 7. & 7. & 6 & $6.825=$ & $5.9-9>3$ & 5 & & \\
\hline orecimiento & $\int C_{a l i f i c z c i o n} 3$ & 5. & & & & J. & 5.1 & & & \\
\hline \multirow{4}{*}{$\begin{array}{c}\text { anuale en } \\
\varnothing\end{array}$} & | Calificacion 4 & 3. & 4. & 4. & & 3. 6 & $4.0 \quad-5$ & 3. & & \\
\hline & | Tota & & 4. & 4. & 4 & 4. $\quad$ S & $4.4>2>3$ & 4. & & \\
\hline & & & 1 & 1. & 2 & 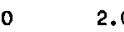 & 2.0 & 2. & & \\
\hline & Total, clases 1- & 3. & 3. $2>>$ & 3. & 3 & 3. & $3.4>2>3$ & 3. $\quad>>>$ & & \\
\hline
\end{tabular}

a El censo de 1950 excluye trabajadores entre las edades 8 y 11 y el censo de 1960 sí los incluye. Debido a
la no comparabilidad se sobrestima la tasa de crecimiento de $1950-1960$ en la categoria 5 . Clave de categorías de calificación: $s=1$ Ingenieros $\mathrm{y}$ científicos; $s=2$ Otros trabajadores técnicos y pro-
fesionales; $s=3$ Empleados administrativos y de oficina; $s=4$ Trabajadores manuales y vendedores no agrícolas; $s=5$ Trabajadores agrícolas no calificados. 
Cuadro 2

MÉXICo: DistribuCtón DE LA FUERZa de TRABaJo POR SECTOR Y CATEGORÍa DE CALIFICACIÓN, 1960

(Miles de años-hombre de la categoría $s$ )

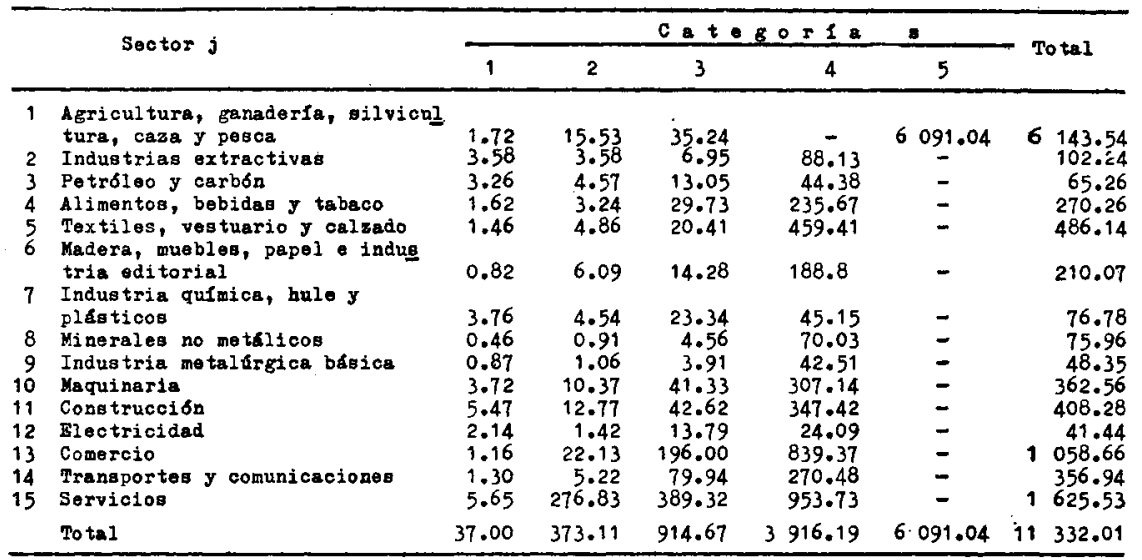

Los coeficientes $l_{s j}^{\tau}$, el insumo de fuerza de trabajo por unidad de capacidad de la generación $\tau$, han sido calculados en dos pasos sucesivos. Primero, estimamos los coeficientes $c_{s j}$, la fracción que la clase de calificación $s$ constituirá en los requisitos totales de mano de obra para la expansión del sector $j$. Segundo, se ha empleado un modelo de cambio neutro de Harrod para estimar $l_{j}^{\tau}$, los requisitos de mano de obra total por unidad de expansión de la capacidad en el sector $j$. Mediante la mezcla de ambos conjuntos de coeficientes, tenemos entonces

$$
l_{s j}^{\tau}=c_{s j} l_{j}^{\tau}
$$

En el cuadro 4 aparecen los valores numéricos estimados de los coeficientes $c_{s j}$ de composición de calificación. En diez de catorce sectores estos coeficientes son idénticos a la distribución de la fuerza de trabajo estimada para 1968. En los cuatro sectores restantes $(6,8,10$ y 15) los coeficientes son semejantes a los de 1968, pero ligeramente mayores en las primeras dos clases de calificación, y representan una extensión de los cambios supuestamente operantes en estos sectores desde $1960 .^{8}$ Estos coeficientes se basan en el censo mexicano de po-

8 Estos cambios reflejan principalmente cambios en la mezcla del producto de estos cuatro sectores, ocasionados por descensos en la proporción de producto generado en los subsectores que requieren proporciones bajas de mano de obra calificada, en comparación con los subsectores más intensivos de calificación. De este modo, en el sector 6, por ejemplo, la producción de la rama de papel e industrias editoriales ha aumentado más aprisa que la de productos de madera; en el sector 10 la producción de automóviles y maquinaria ha crecido más aprisa que la de talleres y servicios de reparación; y en el sector 15, la educación y los servicios profesionales han aumentado más rápidamente que los servicios domésticos o de lavanderia. 
Cuadro 3

México: Distribución ḋe la fUERZa DE TRABajo POR SECTORES Y CATEGoRía DE CALIFICACIÓN, 1968

(Miles de años-hombre de la categoría $s$ )

\begin{tabular}{|c|c|c|c|c|c|c|c|}
\hline & \multirow{2}{*}{ Sector j } & \multicolumn{5}{|c|}{ Categorle 8} & \multirow[b]{2}{*}{ Total } \\
\hline & & 1 & 2 & 3 & 4 & 5 & \\
\hline \multirow[t]{2}{*}{$\begin{array}{r}1 \\
2 \\
3 \\
4 \\
5 \\
6 \\
7 \\
8 \\
9 \\
10 \\
11 \\
12 \\
13 \\
14 \\
15\end{array}$} & $\begin{array}{l}\text { Agricul tura } \\
\text { Minerfa } \\
\text { Potroloo } \\
\text { Alimentos } \\
\text { Textiles } \\
\text { Madera } \\
\text { Qufmica } \\
\text { Minerales no metallicos } \\
\text { Metalúgica bseica } \\
\text { Maquinaria } \\
\text { Construcoion } \\
\text { Electrictlad } \\
\text { Comercio } \\
\text { Transportes } \\
\text { Servicios }\end{array}$ & $\begin{array}{r}5.41 \\
3.76 \\
4.37 \\
2.38 \\
1.89 \\
1.43 \\
5.85 \\
1.05 \\
1.52 \\
13.57 \\
9.16 \\
3.94 \\
2.20 \\
1.82 \\
13.65\end{array}$ & $\begin{array}{r}27.05 \\
3.76 \\
6.11 \\
5.10 \\
6.31 \\
10.61 \\
7.04 \\
2.10 \\
2.28 \\
28.37 \\
21.85 \\
2.58 \\
30.02 \\
6.84 \\
485.98\end{array}$ & $\begin{array}{r}62.22 \\
7.30 \\
17.46 \\
37.42 \\
26.50 \\
21.22 \\
36.29 \\
8.76 \\
7.18 \\
78.31 \\
73.32 \\
25.23 \\
297.30 \\
102.12 \\
581.37\end{array}$ & $\begin{array}{r}9 \overline{9} .49 \\
59.37 \\
295.28 \\
596.32 \\
253.44 \\
70.20 \\
104.84 \\
73.47 \\
496.40 \\
600.63 \\
44.02 \\
1135.01 \\
1345.11 \\
1289.42\end{array}$ & $\begin{array}{c}7024.00 \\
= \\
= \\
= \\
= \\
= \\
=\end{array}$ & $\begin{array}{r}7118.68 \\
107.31 \\
87.31 \\
340.18 \\
631.02 \\
286.70 \\
119.38 \\
116.75 \\
84.45 \\
616.65 \\
704.96 \\
75.77 \\
1464.53 \\
455.89 \\
2370.42\end{array}$ \\
\hline & Total & 72.00 & 646.00 & 1382.00 & 5456.00 & 7024.00 & 14580.00 \\
\hline
\end{tabular}

blación y en las muestras censales de 1960 junto con coeficientes semejantes de los Estados Unidos y otros países industriales avanzados. ${ }^{9}$

Debido a la manera en que se derivaron estos caeficientes, sus sesgos globales van probablemente en la misma dirección que cualesquiera sesgos contenidos en nuestros supuestos acerca de la expansión de las clases de calificación 1-3. Si, por ejemplo, se ha sobrestimado la expansión de la oferta de mano de obra de la clase 3 , entonces otro tanto ha sucedido con los coeficientes de calificación de la demanda de mano de obra de la clase 3 . Este rasgo reduce la sensibilidad del modelo ante errores en las estimaciones correspondientes a los años desde el censo de 1960.

En la estimación de las normas de mano de obra $l_{j}^{\tau}$, empleamos la hipótesis de Harrod sobre cambio tecnológico neutro incorporado, es decir $l_{j}^{\tau}=l_{j} e^{-\rho \tau}$, en donde $l_{j}$ denota la norma de incremento de trabajo que se mantuvo en 1968 , y $\rho$ denota la tasa de crecimiento de la productividad incorporada en años subsecuentes. En lo que sigue, para simplificar la notación y denotar un parámetro de tiempo continuo, omitimos el subíndice sectorial $j$, y reemplazamos el símbolo $l_{j}^{\tau}$ por $l(\tau)$. Para estimar $l(\tau)$, suponemos entonces que las adiciones de capacidad en el sector $j$ han ocurrido a una tasa $\gamma$ sostenida durante el pasado, y que la capacidad ha sido retirada después de una vida de servicio constante $T$. Con estos supuestos, resulta que $\rho$ (la tasa de crecimiento de la productividad de las generaciones nuevas) puede ser

9 Ya en 1960, la composición de la calificación en México, en un gran número de sectores, era comparable a la de los países avanzados. Pero debe reconocerse que las semejanzas, en especial en 1968, se deben en parte a nuestra manera de utilizar las composiciones de calificación de los países industriales como límites superiores de guía para el caso de México, o en los casos en que los datos de este país son incompletos o requieran revisiones posteriores. En relación con las semejanzas en los coeficientes de calificación en distintos países, ver Horowitz et al. (1966) y Keesing (1971). 


\section{Cuadro 4}

MÉXICO: COEFICIENTES $c_{s j}$ MARGINALES DE COMPOSICIÓN DE CALIFICACIÓN (Sectores no agrícolas; después de 1968)

\begin{tabular}{|c|c|c|c|c|c|}
\hline \multirow{2}{*}{\multicolumn{2}{|c|}{ Sector $j$}} & \multicolumn{4}{|c|}{ catagoria s } \\
\hline & & $\begin{array}{c}1 \\
c_{2 j}\end{array}$ & $\begin{array}{r}2 \\
c_{2 j} \\
\end{array}$ & $\begin{array}{c}3 \\
c_{3 j} \\
\end{array}$ & $\begin{array}{c}4 \\
c_{4 j} \\
\end{array}$ \\
\hline $\begin{array}{l}2 \\
3 \\
4 \\
5 \\
6 \\
7 \\
8 \\
9 \\
10 \\
11 \\
12 \\
13 \\
14 \\
15\end{array}$ & $\begin{array}{l}\text { Mineria } \\
\text { Petroleo } \\
\text { Alimentos } \\
\text { Textiles } \\
\text { Madera } \\
\text { Química } \\
\text { Minerales ro metslicos } \\
\text { - Metalúrgica básica } \\
\text { Maquinaria } \\
\text { Construcción } \\
\text { Electricidad } \\
\text { Comercio } \\
\text { Transportes } \\
\text { Servicios }\end{array}$ & $\begin{array}{l}.035 \\
.050 \\
.007 \\
.003 \\
.006 \\
.049 \\
.012 \\
.018 \\
.035 \\
.013 \\
.052 \\
.002 \\
.004 \\
.008\end{array}$ & $\begin{array}{l}.035 \\
.070 \\
.015 \\
.010 \\
.045 \\
.059 \\
.024 \\
.027 \\
.060 \\
.031 \\
.034 \\
.020 \\
.015 \\
.240\end{array}$ & $\begin{array}{l}.068 \\
.200 \\
.110 \\
.042 \\
.085 \\
.304 \\
.090 \\
.085 \\
.140 \\
.104 \\
.333 \\
.220 \\
.224 \\
.250\end{array}$ & $\begin{array}{l}.862 \\
.680 \\
.868 \\
.945 \\
.864 \\
.588 \\
.874 \\
.870 \\
.765 \\
.852 \\
.581 \\
.758 \\
.757 \\
.502\end{array}$ \\
\hline
\end{tabular}

inferida del crecimiento pasado observado de la productividad media. Para probar esto, denotamos los incrementos de la capacidad en el tiempo $t$ mediante $v(t)=v e^{\gamma_{t}}$. Hacemos luego los cálculos siguientes:

Mano de obra requerida en el tiempo $t=$

$$
\begin{aligned}
=L(t) & =\int_{t-T}^{t}\left(l e^{-\rho \tau}\right)\left(v e^{\gamma \tau}\right) d \tau \\
& =\frac{l v}{(\gamma-\rho)}\left[1-e^{(\rho-\gamma) T}\right] e^{(\gamma-\rho) t}
\end{aligned}
$$

Producción obtenida de la capacidad utilizada en el año $t=$

$$
\begin{aligned}
& =X(t)=\int_{t-T}^{t} v e^{\gamma \tau} d \tau \\
& =\frac{v}{\gamma}\left[1-e^{-\gamma T}\right] e^{\gamma t} \\
\therefore \quad \frac{L(t)}{X(t)} & =\frac{L(0)}{X(0)} e^{-\rho t}
\end{aligned}
$$

La ecuación (1) nos permite estimar que la tasa de crecimiento de la productividad es idéntica en la generación promedio en uso en el tiempo $t$, y en la nueva generación que comienza a utilizarse en esa fecha.

Para inferir $l$ a partir de la cantidad observable $L(0) / X(0)$, notamos que: 


$$
\begin{aligned}
\frac{L(0)}{X(0)} & =\frac{l \gamma}{(\gamma-\rho)}\left[\frac{1-e^{(\rho-\gamma) T}}{1-e^{-\gamma T}}\right] \\
\therefore \quad & t=\frac{L(0)}{X(0)}[\theta]
\end{aligned}
$$

en la cual la cantidad $\theta$ se aefine como:

$$
\theta=\frac{\gamma-\rho}{\gamma}\left[\frac{1-e^{-\gamma T}}{1-e^{(\rho-\gamma) T}}\right]
$$

A partir de (3), puede verse que cuando $\rho=0, \theta=1$, de modo que las normas de trabajo marginal y promedio son idénticas. Cuando $T \rightarrow \infty, \theta=\left[\frac{\gamma-\rho}{\gamma}\right] ; y$ cuando escogemos valores típicos para México ( $T=40$ años, $\gamma=7 \%$ anual, y $\rho=3 \%$ anual $)$, el factor $\theta=0.67$. Es decir, para estos valores típicos de los parámetros, la norma de insumo marginal de trabajo será $67 \%$ de la norma promedio observada en el tiempo $t$. Otros cálculos sugieren que estos resultados no se verían seriamente afectados si la vida $T$ de servicio se estableciera a un nivel tan bajo como 30 o tan alto como 50 años.

El cuadro 5 especifica los valores numéricos empleados para $\gamma$,

Cuadro 5

México: Elementos para el cálculo de las normas marginales de TRABAJO DESPUÉS DE 1968

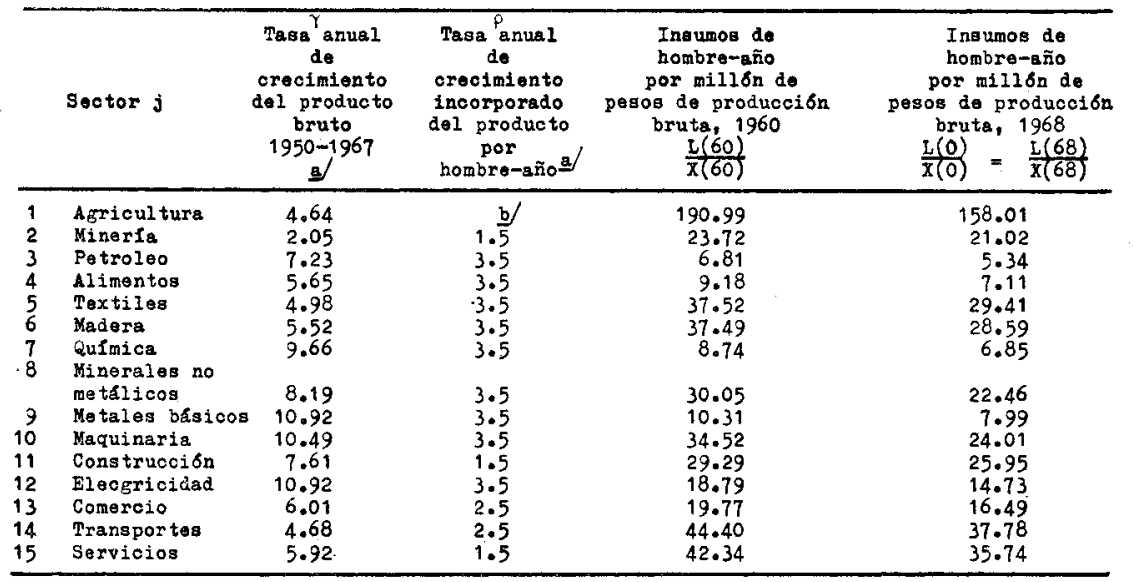

a Tasa anual de crecimiento, compuesta continuamente (porciento).

b Véase el cuadro 6 en cuanto a la formulación de técnicas de producción agrícola alternativas. 
$\rho$ y $L(0) / X(0)$. Para todos los sectores, la vida de servicio $T$ es igual a 40 años.

\section{SUSTITUCIÓN ENTRE ACTIVIdAdES DE PRODUCCIÓN ALTERNATIVAS EN LA AGRICULTURA}

Simultáneamente a la elaboración de DINÁMICO, Barraza, Duloy y Norton comenzaron a trabajar en un modelo de análisis de proceso para el sector agrícola de México. Su formulación de programación lineal incluye actividades alternativas para la sustitución entre capital y trabajo así como patrones de elección de cosechas, sustitución de la demanda, y restricciones sobre la disponibilidad de tierra, trabajo y agua en distritos individuales de irrigación y en áreas de temporal. Una versión del modelo con períodos de un año incluye unas 1000 hileras. En una fecha futura se espera que DINÁMICO pueda ligarse directamente a este modelo sectorial; mientras tanto, con base en datos históricos escasos, hemos intentado tomar en cuenta una elección tecnológica en la agricultura. Los vectores presentes han de considerarse como primera aproximación y serán reemplazados en cuanto se cuente con información mejorada del modelo Barraza-DuloyNorton para el sector agrícola.

La sustitución entre factores (es decir, entre capital y trabajo no calificado) se incluye a través de una elección entre cuatro actividades alternativas. Las alternativas cubren una gama muy amplia de métodos de producción. Éstos se definen como: agricultura mecanizada, moderna, tradicional y de subsistencia, designadas con los subíndices $K, M, T$, y $S$, respectivamente (es decir, en la actividad $X_{1 M}^{t}, X$ se refiere a la producción bruta, $t$ al espacio de tiempo, 1 al sector agrícola, y $M$ a la técnica moderna de producción).

En la construcción de estas cuatro actividades, nuestro método ha sido, primero, proyectar los coeficientes de la agricultura tradicional y moderna con base en datos históricos de áreas geográficas individuales. Los coeficientes de una actividad agrícola mecanizada fueron extrapolados luego a partir de los de la agricultura moderna, reemplazando la mayor parte de la mano de obra no calificada por insumos de capital. Se considera que la actividad agrícola de subsistencia no requiere otro insumo que el trabajo no calificado, y este coeficiente de insumo fue estimado en forma independiente.

La clasificación de los datos observados en dos subsectores (tradicional y moderno) se basó en un análisis de los censos agrícolas de 1950 y 1960.10 Se depositó gran confianza en un estudio de dualidad dentro de la agricultura, elaborado por Trejo y asociados. Este estudio clasifica los municipios en los dos tipos de agricultura - según que sus cocientes capital/trabajo y producción/tierra estén por encima o por debajo del promedio nacional. Con esta base, en 1960 hubo más municipios clasificados como "modernos" que en 1950.

Hay varios riesgos en el uso de estos datos para calcular actividades alternativas. En efecto, suponemos que todos los municipios

10 Ver en especial el estudio del Banco de México (1970a) de Trejo y asociados, Eckstein (1970), Barraza (1969) y Reynolds (1970). 
pueden emplear tecnologías idénticas y que se usan técnicas diferentes debido a las diferencias geográficas en los salarios. Este supuesto es idéntico al empleado por Arrow, Chenery, Minhas y Solow (1961) para inferir una función producción agregativa a partir de datos interna. cionales transversales de los salarios y la productividad del trabajo.

Dentro de las zonas mexicanas dominadas ahora por la agricultura tradicional, no resulta muy claro que la elección óptima de técnicas consista en adoptar mezclas de insumos similares a las observadas en regiones ya convertidas a la agricultura moderna. Muchas de estas últimas tienen posibilidades de riego favorables, terreno nivelado, suelos no degradados y otras ventajas naturales. Los distritos caracterizados como "tradicionales" son a menudo zonas montañosas y densamente pobladas, con suelos desgastados y muy pocas posibilidades de riego no explotadas. Para lograr producción adicional en estas áreas, puede requerirse más trabajo, más capital y todo un paquete de insumos diferentes. Esto sugiere que el crecimiento del producto agrícola continuará concentrándose en los distritos más progresistas, y que puede no ser posible liberar mano de obra de la agricultura tradicional al ritmo que se postula en DINÁMICO.

Pueden mencionarse otros riesgos en nuestro procedimiento. Primero, a juzgar por otras fuentes de información comparable, existen inexactitudes sustanciales en los datos agrícolas censales. Segundo, existe una componente de agricultura de subsistencia en la agricultura "tradicional". Esto conduce a una sobrestimación de los requisitos de fuerza de trabajo y una subestimación de la eficiencia económica de esta actividad. Una dificultad final es que en la agricultura mexicana tanto la productividad del trabajo como la del capital han estado elevándose desde 1960. No se tienen muchas bases sobre las cuales extrapolar estos aumentos o asignarlos entre la agricultura tradicional y la moderna.

En vista de que nuestras estimaciones son burdas, no pareció haber justificación especial para tratar de tomar en cuenta el crecimiento incorporado o desincorporado de la productividad en los coeficientes de insumo del sector. Para obtener estos coeficientes, estimamos que la agricultura moderna en 1967 y 1968 produjo el $60 \%$ del producto del sector, utilizando como insumos el $30 \%$ de la fuerza de trabajo agrícola de México (incluyendo todas las clases de trabajo 1,2 y 3 ), el $61 \%$ del capital privado, el $95 \%$ del capital público, y entre 60 y $100 \%$ de los distintos insumos intermedios. ${ }^{11}$ Luego, redujimos en un $10 \%$ los coeficientes de trabajo y capital resultantes para

11 En comparación, con base en los patrones de producción geográficos, Trejo y asociados [Banco de México (1970 a), cuadro 5] han estimado las participaciones de la agricultura moderna en 1950 y 1960 que se muestran en el cuadro siguiente:

\begin{tabular}{lll}
\hline & 1950 & 1960 \\
\hline Producción bruta & 37.6 & 51.1 \\
Trabajo & 17.8 & 25.0 \\
Capital & 41.0 & 53.5 \\
\hline
\end{tabular}


Cuadro 6

MéXico: Coeficientes Marginales de insumo en cuatro aCtividades AGRÍCOLAS ALTERNATIVAS

(Por millón de pesos de producción agrícola bruta, a precios de 1960)

\begin{tabular}{|c|c|c|c|c|}
\hline & $\frac{1 \mathrm{X}}{\text { Mecanizada }}$ & $\frac{1 M}{\text { Moderna }}$ & $\frac{1 T}{\text { Tradicional }}$ & $\frac{1 \mathrm{~S}}{\text { Subsistencia }}$ \\
\hline 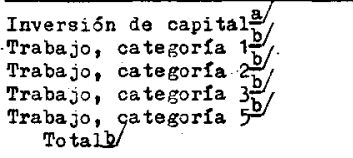 & $\begin{array}{r}4.8 \\
0.20 \\
1.00 \\
2.30 \\
6.50 \\
10.00\end{array}$ & $\begin{array}{r}2.4 \\
0.20 \\
1.00 \\
2.30 \\
66.50 \\
70.00\end{array}$ & $\begin{array}{c}1.5 \\
0 \\
0 \\
0 \\
250.00 \\
250.00\end{array}$ & $\begin{array}{c}0 \\
0 \\
0 \\
0 \\
833.33 \\
833.33\end{array}$ \\
\hline $\begin{array}{l}\text { Costo de los insumos } \\
\text { intermediosa/ } \\
\text { Costo total de los insumos } \\
\text { por cantidad de produccion }\end{array}$ & $\begin{array}{r}.608 \\
1.438\end{array}$ & $\begin{array}{r}.608 \\
1.222\end{array}$ & $\begin{array}{r}.243 \\
1.068\end{array}$ & $\begin{array}{c}0 \\
2.000\end{array}$ \\
\hline
\end{tabular}

a Millones de pesos.

b Hombre-año.

c Valuado el capital al $15 \%$ anual, el trabajo a los salarios de 1960 (cuadro 9) y los insumos intermedios a precios de 1960 .

dar lugar a los cambios tecnológicos que al parecer han economizado ambos insumos. 12

La agricultura mecanizada se considera idéntica a la agricultura moderna en todos los aspectos, excepto que la maquinaria reemplaza la mayoría del trabajo no calificado a un costo de inversión de 40000 pesos por persona (a precios de 1960). ${ }^{13}$ La agricultura de subsistencia es simplificada suponiendo que la adición de un trabajador de la clase 5 resulta en un aumento del producto agrícola de 1200 pesos (equivalentes a alrededor de la mitad de las percepciones de un trabajador agrícola no calificado en 1960), sin insumos adicionales de cualquier otro factor. En el cuadro 6 aparece un resumen de los coeficientes de trabajo y capital resultantes en las cuatro actividades agrícolas. Nótese que a precios de 1960 en la actividad tradicional el costo total de los insumos por unidad de producción es menor que en la moderna. Lo contrario sería válido si todos los demás precios permanecieran constantes y los salarios de la mano de obra no calificada se elevaran de 2400 a 3300 pesos por año-hombre.

12 Nuestras estimaciones no corregidas de los coeficientes de trabajo y capital (por millón de pesos de producción agrícola bruta, a precios de 1960) en los dos subsectores son las siguientes:

\begin{tabular}{lrrrrr}
\hline & \multicolumn{2}{c}{ Moderna } & & \multicolumn{2}{c}{ Tradicional } \\
\cline { 2 - 5 } & 1960 & 1968 & & 1960 & 1968 \\
\hline $\begin{array}{l}\text { Inversión de capital } \\
\text { (millones de pesos) }\end{array}$ & 2.91 & 2.58 & & 1.59 & 1.54 \\
Años-hombre de trabajo & 93.40 & 79.10 & & 294.10 & 277.20 \\
\hline
\end{tabular}

13 Esto significaría que si las tasas de salarios del trabajo agrícola no calificado se elevaron por encima de 6000 pesos por año (a precios de 1960), la mecanización se haría más productiva a un costo del capital de $15 \%$ al año. $(6000 / .15=$ $=40000$ ). 
A diferencia de los otros sectores permitimos que las reubicaciones dentro de la agricultura alteren el patrón de insumos y productos del año base. Se supone que la producción en la agricultura tradicional, estimada en 18000 millones de pesos en 1968, pueda decrecer a partir de entonces, pero que no puede disminuir en más de mil millones de pesos en cualquier año. Esto implicaría operar la actividad de inversión en la agricultura tradicional a una intensidad negativa, liberando 250000 trabajadores de la clase 5 y 1500 millones de pesos de inver. sión por año para transferirla a la agricultura moderna o mecanizada. Así, la fuerza de trabajo en la agricultura tradicional podría disminuir de 5 millones en 1968 a 2 millones en 1980, si la demanda efectiva de mano de obra no calificada permaneciera con suficiente fuerza como para sostener tal flujo hacia afuera.

En vista de las incertidumbres en la construcción de los coeficientes en el sector agrícola, DINAMICO ha sido reprocesado elevando los coeficientes de insumo de trabajo y capital en $50 \%$ en las actividades moderna y mecanizada, pero dejando inalterados los coeficientes de la agricultura tradicional y de subsistencia. Los efectos macroeconómicos de este cambio son sustanciales. En lugar de $7.0 \%$, la tasa anual promedio en 1968-1980 de crecimiento del producto interno bruto desciende a $6.3 \%$. En correspondencia, se reduce la tasa de transferencia rural-urbana. No obstante, continúa siendo óptimo situar la actividad agrícola de subsistencia a una intensidad nula, y reducir la agricultura tradicional a la tasa máxima postulada; la nueva capacidad en la agricultura se dividiría aún entre las actividades moderna y mecanizada. ${ }^{14}$ No hemos encontrado un caso en el cual sea óptimo emplear la técnica de producción de subsistencia.

\section{ACtividades EDUCATIVAS; ESTRUCTURA SALARIAL DE EQUILIBRIO DE VON NEUMAN}

Para expandir la oferta de trabajo más allá de las cifras ya especificadas exógenamente en el cuadro 1, se supone que existen actividades endógenas de formación de capital. $E D_{s}^{t}$ denota la actividad educativa (formación de capital humano) para producir trabajo de calificación $s$ que está disponible por primera vez en el período $t$. Existe una actividad de tal tipo para cada una de las tres categorías de trabajo superiores $(s=1,2,3)$.

Al estimar los coeficientes de estas actividades se ha hecho caso omiso del espacio de salones de clase y otros insumos físicos. Se supone que el insumo principal es el costo de oportunidad del tiempo de trabajo, en la forma de percepciones perdidas por los estudiantes más el tiempo de los maestros y el personal educativo auxiliar. No se ha puesto una restricción presupuestal explícita sobre los gastos educativos. Las tres actividades representan una versión muy simplificada de los procesos de formación de capital humano en México.

14 Un hallazgo similar sería probablemente válido si desagregáramos cualquier otro sector caracterizado por una baja productividad de la fuerza de trabajo. Por ejemplo, si el sector comercio de DINAMICO se desagregara de modo de distinguir entre las técnicas modernas y tradicionales de venta al menudeo, también en este caso probablemente sería óptimo sustituir fuerza de trabajo por capital. 
Educación técnica superior ( $E D_{1}^{t}$ ) requiere seis años (dos períodos) y produce fuerza de trabajo de la clase 1, más un subproducto del trabajo de la clase 2 , a través de los insumos de trabajo de las clases 1 y 2 (tales como profesores) y trabajo de clase 3 (tales como estudiantes ). Debido al rezago de dos períodos, esta actividad está disponible sólo para $t=3,4,5$.

Otra educación profesional ( $E D_{2}^{t}$ ) produce fuerza de trabajo de la clase 2 en tres años (un período de tiempo) con insumos de trabajo de las clases 1 y 2 (tales como maestros) y de la clase 3 (tales como estudiantes ).

Educación secundaria $\left(E D_{3}^{t}\right.$ ) produce fuerza de trabajo de la clase 3 en tres años con insumos de las clases de trabajo 2 y 3 (tales como maestros y personal auxiliar) y trabajo de la clase 4 (estudiantes). Dado el número creciente de aquellos que han terminado la educación primaria es razonable suponer que de entre la clase 4 (urbanos no calificados) no habrá dificultad para encontrar candidatos calificados para la educación secundaria.

Cuadro 7

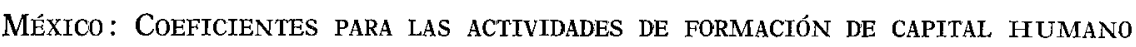

\begin{tabular}{|c|c|c|c|}
\hline \multirow[b]{2}{*}{ Restriccional } & \multicolumn{3}{|c|}{ Incognita } \\
\hline & $\begin{array}{c}\operatorname{ED}_{1}^{t} \\
(t=3,4,5)\end{array}$ & $(t=2,3,4,5)$ & $\begin{array}{c}E D_{3}^{t} \\
(t=2,3,4,5)\end{array}$ \\
\hline $\begin{array}{l}\text { D1, t }-2 \\
\text { D2, t }-2 \\
\text { D3, t }-2 \\
\text { D4, t }-2 \\
\text { D1, t }-1 \\
\text { D2, t }-1 \\
\text { D3, t }-1 \\
\text { D4, t }-1\end{array}$ & $\begin{array}{l}-0.17 \\
-0.09 \\
-2.00 \\
-0.14 \\
-1.40\end{array}$ & $\begin{array}{l}-0.02 \\
-0.18 \\
-1.90\end{array}$ & $\begin{array}{l}-0.16 \\
-0.04 \\
-2.00\end{array}$ \\
\hline $\begin{array}{l}\mathrm{D} 1, t \\
\mathrm{D} 2, t \\
\mathrm{D} 3, t \\
\mathrm{D} 4, t \\
\mathrm{D} 1, t+1 \\
\mathrm{D} 2, t+1 \\
\mathrm{D} 3, t+1 \\
\mathrm{D} 4, t+1\end{array}$ & $\begin{array}{r}1.000 \\
-0.30 \\
-1.30 \\
1.00 \\
0.30 \\
-1.30 \\
. \\
.\end{array}$ & $\begin{array}{r}1.00 \\
-1.00 \\
1.00 \\
-1.00 \\
\vdots \\
\vdots\end{array}$ & $\begin{array}{r}1.00 \\
-1.00 \\
1.00 \\
-1.00 \\
:\end{array}$ \\
\hline
\end{tabular}

a La restricción $D_{s, t}$ se refiere a la oferta total de trabajo de la categoría $s$ en el período $t$

Los coeficientes asignados a las actividades educativas aparecen resumidos en el cuadro 7 . Puede verse que inicialmente deben inscribirse 2 estudiantes para convertir uno en una categoría de mayor calificación en períodos subsecuentes. Los cocientes de estudiantes por escuela y los de desperdicio han sido estimados a través de las estadísticas educativas mexicanas de los años de 1964 a 1968 . El ciclo postsecundario de tres años de "otras personas técnicas y profesionales" es una aproximación burda a una media ponderada global de esta clase de trabajo. 
Una vez que una persona ha entrado en una categoría $s$ en el período $t$, se supone que permanece en esa categoría durante los períodos $t+1, t+2, \ldots$ Debido al horizonte de tiempo comparativaimente corto (12 años entre 1968 y 1980), estas actividades no incluyen consideraciones sobre salidas de la fuerza de trabajo debidas a defunción u otras causas naturales. A la inversa, no se toma en cuenta el aprendizaje en el trabajo, que es un factor que contrarresta la no participación natural en la fuerza de trabajo durante los años inmediatamente siguientes a la educación formal.

A manera de verificación independiente de las normas educativas que aparecen en el cuadro 7, hemos calculado los precios de Von Neumann -suponiendo que se conoce la tasa de rendimiento del capital humano, y que las tasas de salarios relativos de las distintas categorías de calificación permanecerán constantes en un futuro indefinido. Denótese el precio de la calificación $s$ en el tiempo $t$ mediante $p_{s}^{t}$ y tómese la calificación 4 en el tiempo 0 como numerario. Entonces $p_{4}^{0}=1.0$. Las incógnitas son $p_{1,}^{0} p_{2}^{0}$ y $p_{3}^{0}$. Todos los otros precios del trabajo pueden calcularse haciendo $P_{s}^{t}=\beta^{t} P_{s}^{0}$ donde $\beta$ es el factor de actualización, a tres años, de la tasa de rendimiento del capital humano. Para encontrar las tres incógnitas $p_{s}^{0}$, aplicamos las tres ecuaciones simultáneas que garantizan que el costo descontado de producir cada calificación será igual a los beneficios descontados. Por ejemplo, en el caso de la calificación 3 , recordando que $p_{4}^{0}=1.0$ :

$$
\begin{aligned}
& \text { beneficios } \\
& \text { descontados }
\end{aligned}
$$

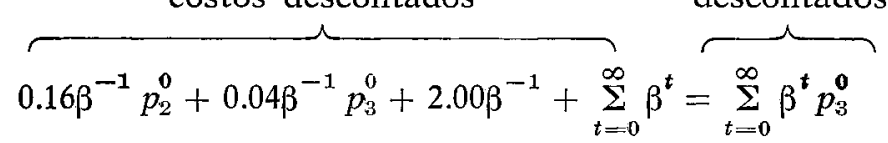

Para resolver las tres ecuaciones simultáneas, debemos especificar un valor particular $r$ de la tasa anual de rendimiento del capital humano. Entonces, en vista de que $\beta$ denota un factor trienal de valor presente, $\beta=\frac{1}{(1+r)^{3}}$. En el cuadro 8 aparece la estructura de precios de eficiencia resultantes para $r=8,12, \ldots, 28 \%$ anual. ${ }^{15}$ Nótese que cuanto mayor sea la tasa de recuperación del capital humano, mayor es la desigualdad del ingreso por salarios. Cuando $r=16 \%$ anual (lo cual significaría que las distintas tasas de recuperación del capital humano y físico son semejantes), las diferencias de calificación son del mismo orden de magnitud que las que prevalecieron en México en los años sesenta.

\section{SUSTITUCIÓN ENTRE CLASES DE CALIFICACióN}

La sustitución directa entre calificaciones se incorpora a través de

I5 Cuando $r \geqslant 30 \%$, no existe un conjunto no negativo de precios del trabajo que satisfaga las tres condiciones que garantizan la igualdad entre los costos descontados y los beneficios. 


\section{Cuadro 8}

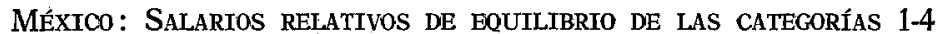
(MODELO DE VON NEUMANN DE FORMaCtóN DE CAPITAL HUMANO TOMANDO COMO BASE LA CATEGORÍA 4)

\begin{tabular}{|c|c|c|c|c|c|c|c|}
\hline & \multicolumn{6}{|c|}{$\begin{array}{c}\text { Tasa de beneficio del capital humano } \\
\text { espocificado exógenamente } \\
\text { I (\% anual). }\end{array}$} & \multirow{2}{*}{$\begin{array}{c}\text { Estimacion gruesa } \\
\text { de los sal arios } \\
\text { anualeg de 1960 } \\
\text { (rolativos a la } \\
\text { categorla 4) }\end{array}$} \\
\hline & 8 & 12 & 16 & 20 & 24 & 28 & \\
\hline \multirow{5}{*}{$\begin{array}{l}\text { Factor trienal de } \\
\text { actualizacion del } \\
\text { capital humano } \\
\text { Salarios do } \\
\text { la categorfa } 1, P_{1}^{0} \\
\text { Salarios de } \\
\text { la categoria } 2, P_{2}^{0} \\
\text { Salarios de } \\
\text { la categorfa } 3, p_{3}^{0} \\
\text { Salarios de } \\
\text { la categoria } 4, P_{4}^{0}\end{array}$} & .7938 & .7117 & .6406 & .5787 & .5244 & .4768 & \\
\hline & 3.42 & 6.28 & 11.88 & $24 \cdot 17$ & 58.14 & 238.67 & 10.00 \\
\hline & 2.59 & 4.08 & 6.51 & 10.94 & 21.08 & 65.83 & 4.17 \\
\hline & 1.64 & 2.11 & 2.77 & 3.84 & 6.09 & 15.43 & 3.00 \\
\hline & 1.00 & 1.00 & 1.00 & 1.00 & 1.00 & 1.00 & 1.00 \\
\hline
\end{tabular}

las actividades de desplazamientos del trabajo a niveles superiores o inferiores. Se supone que el ascenso del trabajo de la clase 5 a la clase 4 , y el descenso del trabajo a la siguiente clase de calificación menor (es decir, de la clase 1 a la 2, de la 2 a la 3, etc.) puede acontecer en cualquier período. En contraste, sólo en los dos primeros períodos (1971 a 1974) se incluye el uso de actividades de ascenso para aumentar el trabajo de las clases 1,2 y 3 y a partir de allí los requisitos de calificación se alcanzan mediante la educación.

En el cuadro 9 se resumen los coeficientes asignados a estas actividades. La sustitución hacia abajo aparece representada por incógnitas no negativas $L D_{s}^{t}(s=1,2,3,4)$ del problema de programación

Cuadro 9

COEFICIENTES DE SUSTITUCIÓN DE CALIFICACIÓN

$$
(t=1,2,3,4,5)
$$

\begin{tabular}{|c|c|c|c|c|c|c|c|c|c|}
\hline \multirow[b]{2}{*}{ Restriccion } & \multirow[b]{2}{*}{ Unidad de medida } & \multicolumn{4}{|c|}{$\begin{array}{l}\text { Aotividades de desplaza } \\
\text { miento niveles inferiores }\end{array}$} & \multicolumn{4}{|c|}{$\begin{array}{l}\text { Aotividades do desplaza } \\
\text { miento a niveles superiores }\end{array}$} \\
\hline & & $L D_{1}^{t}$ & $L D_{2}^{t} \quad L$ & $L D_{3}^{t}$ & $\mathrm{LD}_{4}^{\mathrm{t}}$ & $\mathrm{UL}_{\dagger}^{\mathrm{t}} \mathrm{g} /$ & $\mathrm{UL}_{2^{\mathrm{a}} /}^{t}$ & $\mathrm{OL}_{3^{2}}^{\mathrm{t}}$ & $\mathrm{ur}_{4}^{\mathrm{t}}$ \\
\hline 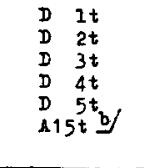 & $\begin{array}{l}\text { Millones de hombres-año } \\
\text { Millones de hombres-zño } \\
\text { Millones he hombres-año } \\
\text { Millones de hombres-año } \\
\text { Millones de hombres-año } \\
\text { Miles de millones de } \\
\text { pesos de } 1960\end{array}$ & $\begin{array}{r}-1.0 \\
1.0\end{array}$ & $\begin{array}{r}-1.0 \\
1.0\end{array}$ & $\begin{array}{r}-1.0 \\
1.0\end{array}$ & $\begin{aligned}-1.0 \\
1.0\end{aligned}$ & $\begin{array}{r}1.0 \\
-1.0 \\
-0.5 \\
-4.0\end{array}$ & $\begin{array}{r}1.0 \\
-1.0 \\
-2.0\end{array}$ & $\begin{array}{r}1.0 \\
-3.0\end{array}$ & $\begin{array}{r}1.0 \\
-1.0 \\
-2.4\end{array}$ \\
\hline \multicolumn{4}{|c|}{ Categorfas de calificacion } & \multicolumn{6}{|c|}{$\begin{array}{c}\text { Estimaciśn gruesa de los salarios } \\
\text { anualos de } 1960 \text { (miles de } \\
\text { pesos por hombromaño) }\end{array}$} \\
\hline \multicolumn{5}{|c|}{$\begin{array}{l}\mathbf{s}=1 \text { Ingenieros y cientificos } \\
\text { = }=2 \text { otros trabajadores técnicos y profesionales } \\
\text { a }=3 \text { Empleados administrativos y de oficina } \\
\text { s }=4 \text { Trabajadores manuales y vendedores no agricolas } \\
\text { s }=5 \text { Trabajadores agricolas no calificados }\end{array}$} & \multicolumn{5}{|c|}{$\begin{array}{r}72.0 \\
30.0 \\
21.6 \\
7.0 \\
2.4\end{array}$} \\
\hline
\end{tabular}

a Las actividades $U L_{s}^{t}(s=1,2,3)$ sirven para representar las opciones de sustitución a corto plazo en las tres categorias de calificación superiores, y por lo tanto están limitadas a los primeros períodos $(t=1,2)$.

b La definición del sector 15 es: servicios, incluyendo el gobierno. 
lineal, definidas como la cantidad de trabajo desplazado a nivel inferior de la categoría de calificación $s$ a la $s+1$ en el período $t$. De este modo, el trabajo de la categoría 1 tiene calificación para desarrollar empleos de la categoría 2, los de la 2 el de la clase 3 , etc.

En la dirección contraria, la transferencia de trabajo de la clase 5 a la 4 correspondería a la emigración de las actividades agrícolas a otras. Parte de la migración se presentaría aun cuando fueran cero las diferencias entre los salarios urbanos y los rurales. Para transferir más trabajo de la agricultura a otras actividades (más allá del caso en que las diferencias salariales fueran cero), y para calificar esta mano de obra para el trabajo en los sectores no agrícolas, hemos postulado la actividad mejorada del trabajo $U L_{4}^{t}$. A través de esta actividad, un trabajador agrícola puede convertirse en un trabajador no agrícola no calificado, en cualquier período, a un costo anual recurrente de 2400 pesos valuados en servicios urbanos (vivienda, salud, educación, servicios bancarios, cines, etc.). ${ }^{16}$ Consideramos aquí los 2400 pesos de servicios como un insumo interindustrial asociado a la conversión de un tipo de trabajo en otro. No se adscribe ninguna utilidad directa a este costo de transformación. Al excluir este flujo de servicios de nuestra definición de consumo agregado, nos alejamos de las convenciones de la contabilidad nacional.

En las actividades que proporcionan sustitución a corto plazo de las tres categorías de calificación superiores $\left(U L_{s}^{t}\right.$ donde $s=1,2,3$ y $t=1,2$ ), todos los insumos consisten en categorías de trabajo de baja calificación. Los coeficientes pueden interpretarse en el sentido de que significan que el personal con adiestramiento adecuado puede ser sustituido por personal de la clase de calificación inferior siguiente, pero que la consecuencia será un descenso de la productividad del resto de la fuerza de trabajo. Por ejemplo, los coeficientes de la actividad $U L_{1}^{t}$ implican que si un ingeniero debe ser reemplazado temporalmente por un técnico, habrá ineficiencias serias en el resto del proceso productivo, y que estas ineficiencias generarán una demanda de una mitad adicional de empleados de oficina y cuatro trabajadores manuales. Los coeficientes de la actividad $U L_{2}^{t}$ significan que si un profesionista, tal como un maestro o un médico, se reemplaza por un sustituto de la clase 3 con adiestramiento inadecuado, la economía perderá como resultado directo o indirecto, el servicio de dos trabajadores de la clase 4.

En conjunto, las actividades resumidas en el cuadro 9, ayudan a establecer límites inferiores y superiores a los coeficientes entre precios de eficiencia de cada clase de mano de obra. Por ejemplo, las actividades $L D_{3}^{t}$ y $U L_{3}^{t}$ juntas, implican que en los primeros dos períodos el salario de eficiencia de un trabajador de oficina debe quedar en algún lugar dentro del intervalo entre una y tres veces el de los trabajadores manuales. De los cocientes de salarios de eficiencia de

16 La cifra de $\$ 2400$ se estimó como la mitad de la diferencia entre los salarios urbanos y los rurales en 1960. Más que suponer que esta diferencia total es gastada en servicios, sería más correcto distribuir esta cantidad también entre otros sectores. 
otras actividades de desplazamiento a nivel superior o inferior pueden deducirse límites semejantes.

\section{Resultados}

En el cuadro 10 aparece la distribución de la calificación de la mano de obra en 1980 por sector de ocupación y en el cuadro 11 aparecen los detalles anuales de los insumos y productos de las actividades de sustitución educativas endógenas y de sustitución de actividades de calificación a corto plazo. Nótese que para todas, salvo una categoría de calificación en un período, la oferta de fuerza de trabajo disponible exógenamente aumenta según la producción de las actividades de formación de capital humano. En cada caso, se emplean las actividades mejoradoras de calificación a corto plazo. (Por ejemplo, en 1980, $t=4$, las áreas urbanas requieren 2332 miles de trabajadores según las cifras postuladas exógenamente.) Sólo en un caso se presenta desplazamiento del trabajo a nivel inferior.

\section{Cuadro 10}

MÉXico: Distribucrón DE LA FUERza DE TRABaJo POR SECTORES Y CATEGORÍAS DE CALIFICACTÓN

(Miles de personas)

\begin{tabular}{|c|c|c|c|c|c|c|c|c|}
\hline \multirow{2}{*}{\multicolumn{2}{|c|}{ Sector $j$}} & \multicolumn{5}{|c|}{$c a t e c \circ \times 1 a$ a } & \multirow{2}{*}{\multicolumn{2}{|c|}{ Total }} \\
\hline & & 1 & 2 & 3 & 4 & 5 & & \\
\hline $\begin{array}{l}1 \\
2 \\
3 \\
4 \\
5 \\
6 \\
7 \\
8\end{array}$ & $\begin{array}{l}\text { Agricul tura } \\
\text { Minerfa } \\
\text { Petroleo } \\
\text { Alimentos } \\
\text { Textiles } \\
\text { Madera } \\
\text { qufmica } \\
\text { Minerales no }\end{array}$ & $\begin{array}{r}14.43 \\
5.86 \\
7.45 \\
3.40 \\
2.72 \\
2.47 \\
16.62\end{array}$ & $\begin{array}{r}72.15 \\
6.11 \\
10.92 \\
7.52 \\
9.38 \\
18.47 \\
21.97\end{array}$ & $\begin{array}{r}165.94 \\
12.29 \\
32.46 \\
56.49 \\
40.64 \\
37.32 \\
117.35\end{array}$ & $\begin{array}{c}- \\
143.21 \\
98.23 \\
415.33 \\
855.65 \\
403.41 \\
183.12\end{array}$ & $\begin{array}{c}6498.17^{9 /} \\
= \\
= \\
= \\
= \\
=\end{array}$ & & $\begin{array}{l}750.69 \\
167.47 \\
149.06 \\
482.74 \\
908.39 \\
461.67 \\
339.06\end{array}$ \\
\hline $\begin{array}{l}9 \\
9 \\
10 \\
11 \\
12 \\
13 \\
14 \\
15\end{array}$ & $\begin{array}{l}\text { metalicos } \\
\text { Metales bábicos } \\
\text { Maquinaria } \\
\text { Construcoion } \\
\text { Eloctricidad } \\
\text { Comercio } \\
\text { Transportes } \\
\text { Servicios }\end{array}$ & $\begin{array}{r}2.30 \\
3.83 \\
42.93 \\
22.57 \\
9.37 \\
4.13 \\
3.40 \\
32.02\end{array}$ & $\begin{array}{r}4.79 \\
6.64 \\
79.61 \\
58.04 \\
6.54 \\
.53 .28 \\
13.54 \\
1034.76\end{array}$ & $\begin{array}{r}18.19 \\
20.39 \\
211.85 \\
203.63 \\
67.42 \\
\\
551.81 \\
209.21 \\
1260.67\end{array}$ & $\begin{array}{r}195.09 \\
181.97 \\
1 \quad 122.15 \\
1454.70 \\
96.87 \\
1865.32 \\
623.48 \\
2 \quad 329.97\end{array}$ & $\begin{array}{l}\bar{z} \\
\bar{z} \\
\bar{z} \\
\overline{-}\end{array}$ & $\begin{array}{l}1 \\
1 \\
2 \\
4\end{array}$ & $\begin{array}{l}220.37 \\
212.83 \\
456.54 \\
738.94 \\
180.20 \\
474.54 \\
849.63 \\
657.42\end{array}$ \\
\hline . & Total & 173.50 & 1403.72 & 3005.66 & 9968.50 & 6498.17 & 21 & 049.55 \\
\hline
\end{tabular}

a Incluye 386000 trabajadores agrícolas no calificados como redundantes. Debido a la restricción de no negatividad sobre la actividad $V_{1 s}^{4}$ no es óptimo emplear estos trabajadores en la actividad agrícola de subsistencia durante 1980 (período 4).

Se recuerda al lector que la representatividad de nuestras fuentes de información es susceptible de mejorarse en forma significativa y que estos resultados detallados no pueden tomarse con demasiado rigor. En particular creemos que DINÁMICO subestima en forma sistemática la demanda de educación intermedia y superior justificada económicamente. Durante el período 1960-1968, México mejoró la educación de su población de manera tan rápida que en 1968 la fuerza de trabajo debió haber tenido mayor educación dentro de cada clase de calificación que en 1960. Ciertos cálculos por separado sugieren 
que los niveles educativos medios de los incrementos de fuerza de trabajo, durante estos años, debieron haber sido dos grados mayores que la ecuación promedio dentro de la misma clase de calificación en 1960. Nuestro modelo reconoce sólo un aspecto de las necesidades educativas futuras: los requisitos de mano de obra calificada adicional ocasionados por el cambio en la estructura del producto, manteniendo en los niveles de 1968 la calidad y la efectividad de los incrementos de la fuerza de trabajo. Se hace caso omiso del otro aspecto de la necesidad de educación, es decir, el papel de la educación en la promoción del crecimiento de la productividad a través del mejoramiento de la calidad de la mano de obra. Este error no puede rectificarse fácilmente, pero conduce a un sesgo hacia abajo de nuestras estimaciones de requisitos de personal calificado, y a un sesgo hacia arriba en cuanto al personal no calificado.

Teniendo en cuenta los requisitos de mejoramiento, los resultados de DINAMICO son ampliamente congruentes con las tasas planeadas de crecimiento de la ecuación media y superior. (La tasa de expansión de las demandas de las categorías de calificación 1-3 es del mismo orden de magnitud que la del producto interno: $7 \%$ por año). Sobre esta base, México no se enfrenta a estrangulamientos cuantitativos graves que se refieran a clases amplias de mano de obra calificada. Una implicación relacionada con esto es que si la educación postprimaria se expande a tasas anuales superiores a $7 \%$, ello conducirá a un aumento creciente de los niveles educativos dentro de cada categoría de empleo. El adiestramiento de baja calidad puede volverse cada vez más arriesgado y antieconómico tanto para el individuo como para la sociedad. Puede esperarse que la demanda efectiva se desplace hacia un elevado adiestramiento y una educación general más elevada de todos los ciudadanos. 
Cuadro 11

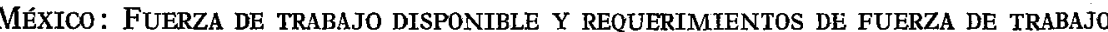

(Se omiten los insumos y los productos cuya intensidad óptima es cero)

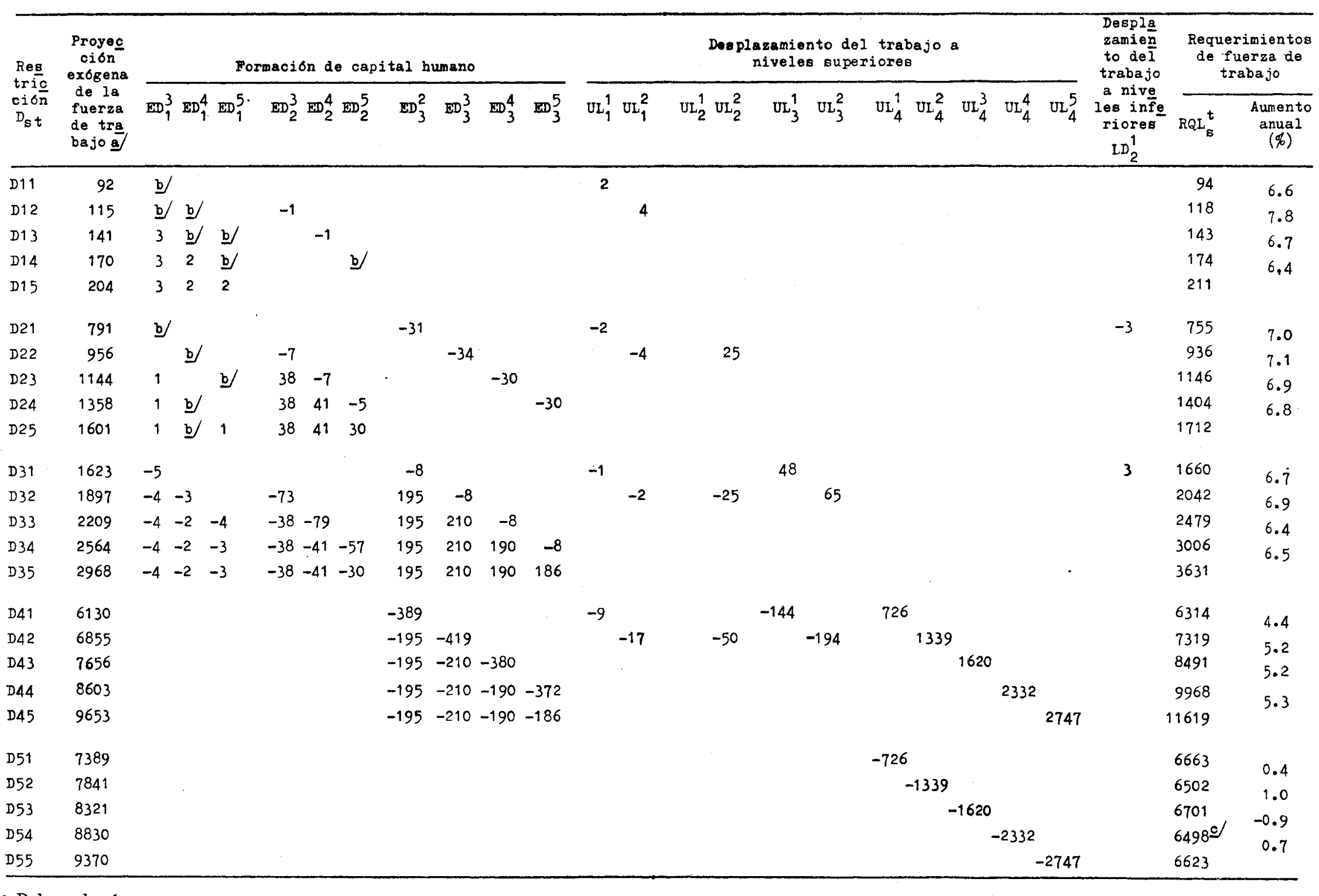

Del cuadro 1 .

Incluye 386000 trabajadores agrícolas no calificados como redundantes. Debido a la restricción de no negatividad sobre la actividad $V_{1 s}^{4}$ no es óptimo emplear estos trabajadores en la actividad agrícola de subsistencia durante 1980 (período 4). 


\section{REFERENCIAS BIBLIOGRAFICAS}

Adelman I., "A Linear Programming Model of Educational Planning: A Case Study of Argentina", en I. Adelman y E. Thorbecke (eds.), The Theory and Design of Economic Development, Baltimore, Johns Hopkins Press, 1966.

Arrow, K. J., H. B. Chenery, B. Minhas, y R. M. Solow, "Capital-Labor Substitution and Economic Efficiency", Review of Economics and Statistics, agosto de 1961.

Banco de México, Cuadro de insumo-producto de México, 1960, diciembre de 1966.

- Encuesta sobre ingresos y gastos familiares en México, 1963, 1966.

- Departamento de Estudios Económicos, Cuentas nacionales y acervos de capital, consolidadas y por tipo de actividad económica, 1950-1967, junio de 1969. - - La dualidad de la agricultura mexicana, estudio dirigido por Saúl Trejo, $1970(\mathrm{a})$.

- - Proyecciones de la economía mexicana para 1976, 1970(b).

- Departamento de Investigaciones Industriales, El empleo de personal técnico en la industria de transformación, 1959.

_. Oficina de Recursos Humanos, y Secretaría Técnica de la Comisión Nacional de Planeamiento Integral de Educación, Información estadística, marzo de 1968.

Barraza, L., "La dualidad en la agricultura mexicana, Revista del Colegio de Economistas de México, 1969.

Benítez Zenteno, R., Análisis demográfico de México, Instituto de Investigaciones Sociales, Universidad Nacional Autónoma de México, 1961.

Benítez Zenteno, R., y G. Cabrera Acevedo, Proyecciones de la población de México, 1960-1980, Banco de México, Depto. de Investigaciones Industriales, Oficina de Recursos Humanos, 1966.

Bowles, S., Planning Educational Systems for Economic Growth, Cambridge, Massachusetts, Harvard University Press, 1969.

Bruno, M., "A Programming Model for Israel", en I. Adelman y E. Thorbecke (eds.), The Theory and Design of Economic Development. Baltimore, Johns Hopkins Press, 1966

Carnoy, M., "Earnings and Schooling in Mexico", Economic Development and Cultural Change, vol. 15, Núm. 4, julio de 1967, pp. 408-419.

-, "Rates of Return to Schooling in Latin America", Journal of Human Resources, vol. II, Núm. 3, verano de 1967, pp. 359-374.

Centro Latinoamericano de Demografía (CELADE), Boletín Demográfico, año 2 , vol. III, enero de 1969 (incluye proyecciones de la población de México y su estructura de edades hasta 1985).

Davis, R. C., Scientific, Engineering and Technical Education in Mexico, Education and World Affairs Occasional Report No. 3, Nueva York, abril de 1967.

Eckstein, S., El marco macroeconómico del problema agrario mexicano, Centro de Investigaciones Agrarias, 1970.

El Colegio de México, Centro de Estudios Económicos y Demográficos, Estudios de población y fuerza de trabajo, con base en una muestra del censo de población en 1960, México, junio de 1966 . Véase también el resumen presentado como "Informe" en DEMOGRAFÍA Y EConomía, vol. II, Núm. 2, 1968

Frank, C. R. Jr., "Urban Unemployment and Economic Growth in Africa", Oxford Economic Papers, vol. 20, Núm. 2, julio de 1968.

Horowitz, M., M. Zymelman e I. L. Herrenstadt, Manpower Requirements for Planning: An International Comparison Approach, 2 vols., Boston, Northeastern University, diciembre de 1966.

Isbister, J., The Growth of Employment in Mexico, tesis inédita, Princeton University, 1969.

- "Urban Employment and Wages in a Developing Economy: The Case of Mexico", versión preliminar inédita, University of California en Santa Cruz, diciembre de 1969.

Keesing, D. B., "Different Countries' Labor Skill Coefficients and the Skill Intensity of International Trade Flows", Journal of International Economics, mayo de 1971.

-, "Structural Change Early in Development: Mexico's Changing Industrial and Occupational Structure from 1895 to $1950^{\circ}$, Journal of Economic History, vol. XXIX, Núm. 4, diciembre de 1969, pp. 716-738.

Lajous Vargas, A., Aspectos de la educación superior y el empleo de profesionis tas en México, 1959-1967, tesis, Escuela Nacional de Economía, Universidad Nacional Autónoma de México, 1967. 
- "Aspectos regionales de la expansión de la educación superior en México, 1959-1967". DEMOGRAFÍA y ECONOMía, vol. II, Núm. 3, 1968, pp. 404-427.

Myers, C. N., Education and National Development in Mexico, Industrial Relations Section, Department of Economics, Princeton University, Princeton, Nueva Jersey, 1965.

Naciones Unidas, "Income Distribution in Latin America", Economic Bulletin for Latin America, vol. 12, octubre de 1967, pp. 38-60.

Navarrete, I. M. de, La distribución del ingreso y el desarrollo económico de México, Instituto de Investigaciones Económicas, Escuela Nacional de Economía, Universidad Nacional Autónoma de México, 1960.

Oshima, H. T., "Gowth and Unemployment in Post War Asia", en Structure and Development in Asian Economies, Japan Economic Research Center, Tokio, diciembre de 1968.

Reynolds, C. W., The Mexican Economy: Twentieth Century Structure and Growth, New Haven, Yale University Press, 1970.

Secretaria de Educación Pública, Dirección General de Enseñanza Superior e Investigación Científica, Concentración estadistica del sistema educativo, septiembre de 1960.

Secretaría de Economía, Dirección Generăl de Estadística, Séptimo Censo General de Población, 6 de Junio de 1950, Resumen General, 1953

- Séptimo Censo General de Población 1950. Parte Especial, 1955.

Secretaría de Industria y Comercio, Dirección General de Estadística, Anuario Estadístico de los Estados Unidos Mexicanos, ediciones de 1941, 1945-50, 1951-52, 1955-56, 1960-61, 1964-65, y 1966-67.

— IV Censos Agricoia-Ganadero y Ejidal 1960. Resumen General, 1965.

- VIII Censo General de Población, 1960. Población Económicamente Activa (Rectificación a los Cuadros 25, 26 y 27 del Resumen General ya Publicado), 1964. , VIII Censo General de Población, 1960. Resumen General, 1962.

- VIII Censo Industrial 1966, Datos de 1965, Resumen General, 1967.

- Dirección General de Muestreo, La población económicamente activa de México en junio de 1964, 3 vols., 1964.

Strassman, W. P., Technological Change and Economic Development, The Manufacturing Experience of Mexico and Puerto Rico, Ithaca, Nueva York, Cornell University Press, 1968.

Tabah, L., "Proyecciones de población activa a través de representaciones matriciales", DEMOGRAFÍA Y ECONOMÍA, vol. II, Núm. 2, 1968, pp. 205-240.

Thorbecke, E., "Unemployment and Underemployment in the Developing World", trabajo presentado a la Conferencia sobre Desarrollo Económico Internacional, Columbia University, Williamsburg, Virginia y Nueva York, febrero 15-21, 1970.

Todaro, M. P., "An Analysis of Indusirialization, Employment and Unemployment in Less Developed Countries", Yale Economic Essays, otoño de 1968.

Unikel, L., "El proceso de urbanización en México: distribución y crecimiento de la población urbana", peMografía y ECONoMía, vol. II, Núm. 2, 1968, pp. 139-182.

U. S. Department of Commerce, Censis of Population 1960, Special Reports on Occupation by Industry, y Residence Overseas.

Universidad Nacional Autónoma de México (UNAM), Anuario Estadistico 1959, 1960.

Urquidi, V. L. y A. Lajous Vargas, Educación superior, ciencia y tecnología en el desarrolto económico de México, México, El Colegio de México, 1967.

Yates, P. Lamartine, El desarrollo regional de México, Banco de México, Depto. de Investigaciones Industriales, 1961.

Wilkie, J. W., The Mexican Revolution: Federal Expenditures and Social Change Since 1910, Berkeley y Los Ángeles, University of California Press, 1967. 